\title{
Noninvasive genetic assessment of the population trend and sex ratio of the Shennongjia population of Sichuan snub-nosed monkeys (Rhinopithecus roxellana)
}

\author{
CHANG ZongFei ${ }^{1,2,3}$, LIU ZhiJin $^{1}$, YANG JingYuan ${ }^{4}$, LI Ming $^{1 *} \&$ VIGILANT Linda ${ }^{2 *}$ \\ ${ }^{1}$ Key Laboratory of Animal Ecology and Conservation Biology, Institute of Zoology, Chinese Academy of Sciences, Beijing 100101, China; \\ ${ }^{2}$ Department of Primatology, Max Planck Institute for Evolutionary Anthropology, Leipzig 04103, Germany; \\ ${ }^{3}$ Graduate University of the Chinese Academy of Sciences, Beijing 100039, China; \\ ${ }^{4}$ Hubei Province Key Laboratory of Conservation Biology of Shennongjia Golden Monkey, Muyu 442411, China
}

Received June 26, 2011; accepted October 21, 2011; published online December 8, 2011

\begin{abstract}
Effective population management relies on assessments of population size and sex ratio. However, these estimates are difficult to obtain for elusive and rare species. Recently, noninvasive genetic census methods have been developed as an alternative to traditional capture-mark-recapture methods. In this study, we estimated the size of the Sichuan snub-nosed monkey (Rhinopithecus roxellana) population in the Shennongjia Nature Reserve (SNR) using a noninvasive sampling method based on 16 microsatellite loci. We also used a PCR-based genetic method to sex the sampled individuals and infer the population sex ratio. The population size of $R$. roxellana in the SNR was estimated to be 1044 individuals (95\% $\mathrm{CI}_{\mathrm{TIRM}}$ : 613-1409). The estimated population sex ratio is more female-biased than expected, which we attribute to the sampling biased towards one male units and limited sampling of bachelor male units. Moreover, there is no suggestion that the heavy traffic road through the reserve might block movement of monkeys. The results of this study indicate genetic assessments based on a noninvasive sampling method can provide useful information regarding populations of elusive primates.
\end{abstract}

snub-nosed monkey, Shennongjia, noninvasive sampling, population size, sex ratio

Citation: Chang Z F, Liu Z J, Yang J Y, et al. Noninvasive genetic assessment of the population trend and sex ratio of the Shennongjia population of Sichuan snub-nosed monkeys (Rhinopithecus roxellana). Chin Sci Bull, 2012, 57: 1135-1141, doi: 10.1007/s11434-011-4872-6

The Sichuan snub-nosed monkey (Rhinopithecus roxellana), also known as the golden monkey, is an endangered primate endemic to China. Despite a wide distribution in China during the Pleistocene [1], wild $R$. roxellana populations now only occur in 3 isolated mountainous regions. The total population size is estimated to number about $15000[2,3]$. Because of large-scale commercial timber exploitation between the 1950 s and early $1980 \mathrm{~s}$, the habitats of $R$. roxella$n a$ in the Shennongjia Nature Reserve (SNR) became fragmented, and illegal hunting led to the capture of at least 130 monkeys in the 1970s and 1980s [4]. In the SNR, the population size was estimated to be more than 2000 individuals prior to 1970 [5]. However, the population had dropped to an

*Corresponding authors (email: lim@ioz.ac.cn; vigilant@eva.mpg.de) estimated 500 individuals in 1989, a reduction of $75 \%$ [4].

Effective conservation management requires data on population size and sex ratio. Traditional capture-markrecapture (CMR) methods are an efficient tool but may be difficult to apply to rare, elusive and capture-sensitive species [6]. In addition, traditional CMR methods that require trapping or handling of individuals may harm the animals. For difficult-to-observe species, noninvasive genetic surveys have in recent years provided valuable information for the management and monitoring of populations [7-13]. These studies showed that use of molecular census techniques may reduce time and effort and provide more detailed information about elusive species [14]. However, because of the relatively high cost, noninvasive genetic census techniques normally are applied only to small areas 
or small and/or isolated populations [11].

$R$. roxellana is highly arboreal and only occasionally descend to the ground. Because of their shy nature, quick movements and occurrence in remote and inaccessible areas, achieving estimates of population size is difficult. Unlike some other ape species, which build large nests that are easily detected, $R$. roxellana are not nest builders. The most commonly used census method is individual counts based on line transects. However, traditional line transect methods do not work well for arboreal primates that tend to flee silently through the forest canopy before being counted [15]. In addition, vegetation can often hamper efforts to spot and count individuals. Cui et al. [16] once estimated group sizes of Yunnan snub-nosed monkeys (Rhinopithecus bieti) on the basis of feces amount at sleeping sites. However, this method gave a highly uncertain estimate owing to the influence of weeds, shrubs and tangles of fallen branches [16].

In this study, we conducted a pilot project aimed at determining the suitability of noninvasive genetic surveys for $R$. roxellana in the SNR. Our goals were to elucidate the population trend during the past two decades and estimate the sex ratio of the wild $R$. roxellana population in the SNR. We also suggest directions for further research and place our results in the context of conservation of this endangered species.

\section{Materials and methods}

\subsection{Study site and sample collection}

Research was conducted in the Shennongjia Nature Reserve $\left(31^{\circ} 21^{\prime} 20^{\prime \prime}-31^{\circ} 36^{\prime} 20^{\prime \prime} \mathrm{N}, 110^{\circ} 03^{\prime} 05^{\prime \prime}-110^{\circ} 33^{\prime} 50^{\prime \prime} \mathrm{E}\right)$ in $\mathrm{Hu}-$ bei Province, China (Figure 1). The SNR is a mountainous area of ca. $705 \mathrm{~km}^{2}$. One road passes through $R$. roxellana habitat, which consequently represents a potential hindrance to monkey movement. The SNR was covered originally with primary forest. A large part of the reserve was clearcut between the 1950s and early 1980s [17]. Presently, 4 types of habitats occur in the reserve: grassland, shrub forest and bamboo, young forest, and primary forest [18].

We collected fecal samples from throughout the study site from November 2007 to October 2008. R. roxellana are diurnal animals. Li [19] showed $R$. roxellana in the SNR exhibit 2 travel peaks (morning and afternoon) and a midday rest period with little or no travel. The rest periods for all seasons were identical, of about $1.5 \mathrm{~h}$ duration from $12: 45$ to $14: 15$. Generally, the monkeys would defecate during rest periods and we could collect the fecal samples under the trees after the monkeys left the rest site. Thus, most of the fecal samples in our study were defecated by the monkeys on the same day, with the exception of the site YJW, which was estimated to be 3 days old. The geographic coordinates of each group sampled were recorded using a GPS. In total, we collected 337 fecal samples at 8 sites (Figure 1), which were desiccated using the two-step ethanol-silica

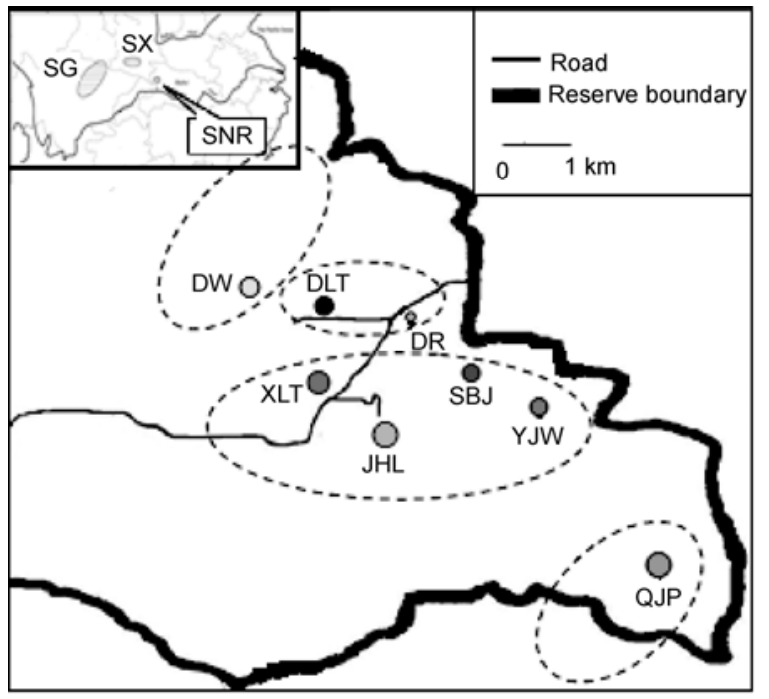

Figure 1 Location of the Shennongjia Nature Reserve study area. Collection sites are indicated by circles with different colors representing different sample periods. The size of the circles is directly proportional to the number of fecal samples collected. Dashed ellipses indicate the seasonal distribution ranges of golden monkeys in the SNR.

procedure [20] and stored at room temperature.

\subsection{DNA extraction and amplification}

Fecal samples were extracted using the QIAmp Stool Kit (QIAGEN). Two negative extraction controls were processed along with 10 fecal extractions. The amount of amplifiable DNA in the extracts was estimated by means of a quantitative polymerase chain reaction (PCR) assay as previously described [21]. Extracts that contained less than 10 pg DNA/ $\mu \mathrm{L}$ were not analyzed further.

Three independent amplifications from each DNA extract were performed for 16 loci originally characterized in humans and used for studies of other primates (D1S533, D1S1656, D1S1665, D2S442, D5S1457, D6S474, D6S493, D6S1056, D7S817, D7S1826, D7S2204, D10S611, D10S676, D10S1432, D13S321 and D14S306) together with a minimum of 6 negative controls (in which $5 \mu \mathrm{L} \mathrm{H}_{2} \mathrm{O}$ rather than DNA was added to the well). A two-step multiplex PCR method was used as described by Arandjelovic et al. [22]. Multiplex PCRs were conducted in a $20-\mu \mathrm{L}$ reaction volume containing $1 \times$ SuperTaq buffer (HT Biotechnology), $1.75 \mathrm{mmol} / \mathrm{L} \mathrm{MgCl}_{2}, 0.15 \mathrm{mmol} / \mathrm{L}$ of each forward (unlabelled) and reverse (unlabelled) primer, $110 \mu \mathrm{mol} / \mathrm{L}$ of each dNTP, $16 \mu \mathrm{g}$ bovine serum albumin, $0.5 \mathrm{U}$ SuperTaq (HT Biotechnology) premixed 2:1 with TaqStart Antibody (BD Biosciences), and $5 \mu \mathrm{L}$ template DNA. The PCR protocol was as follows: initial denaturation for $9 \mathrm{~min}$ at $94^{\circ} \mathrm{C}$, 30 cycles of $20 \mathrm{~s}$ at $94^{\circ} \mathrm{C}, 30 \mathrm{~s}$ at $55^{\circ} \mathrm{C}$, and $30 \mathrm{~s}$ at $72^{\circ} \mathrm{C}$, and a final extension of $5 \mathrm{~min}$ at $72^{\circ} \mathrm{C}$. Singleplex PCRs were carried out as above but with the following modifications: $5 \mu \mathrm{L}$ of $1: 100$ diluted multiplex PCR product was used as template, half the amount of $\mathrm{MgCl}_{2}(0.875 \mathrm{mmol} / \mathrm{L})$ 
was added, and only 0.35 U of SuperTaq (HT Biotechnology) premixed 2:1 with TaqStart Antibody was used. Moreover, each singleplex PCR only contained a single primer pair: $0.25 \mathrm{mmol} / \mathrm{L}$ of a FAM, HEX, or NED fluorescently labeled forward primer and $0.25 \mathrm{mmol} / \mathrm{L}$ of a reverse primer. To guard against contamination, all steps of the PCR set-up for DNA samples (except the addition of template) were performed under a hood that was irradiated with ultraviolet light before and after use.

Up to 3 different PCR products were combined and electrophoresed on an ABI PRISM 3100 Genetic Analyser and allele size was determined relative to an internal size standard (ROX-labeled HD400) using GeneMapper version 3.7 software (Applied Biosystems). Genotypes were confirmed with $99 \%$ certainty by observation of each allele twice in two or more independent reactions for heterozygote genotypes, while homozygous genotypes were ascertained by up to 5 independent observations depending on the quality of DNA in the extract.

\subsection{Sex identification}

Villesen and Fredsted [23] designed a PCR assay to reliably sex ape and monkey DNA samples. The PCR yields fragment sizes of $180 \mathrm{bp}(\mathrm{X})$ and $209 \mathrm{bp}$ (Y), respectively. Males were identified by two bands (the $\mathrm{X}$ and $\mathrm{Y}$ fragments) and females were identified by a single band (the $\mathrm{X}$ fragment). DNA of one known male and one known female monkey from the SNR were used as positive controls. Cycling conditions were $94^{\circ} \mathrm{C}$ for $5 \mathrm{~min}$, and 45 cycles of $94^{\circ} \mathrm{C}$ for $30 \mathrm{~s}, 62^{\circ} \mathrm{C}$ for $30 \mathrm{~s}$ and $72^{\circ} \mathrm{C}$ for $30 \mathrm{~s}$, and a final extension of $72^{\circ} \mathrm{C}$ for $10 \mathrm{~min}$. Each sample was amplified 3 times and PCR fragments were separated on $2.5 \%$ agarose gels $(100 \mathrm{~V}, 1 \mathrm{~h})$. A sample was identified as male if all amplifications showed the $\mathrm{Y}$ band, and as female if no $\mathrm{Y}$ band was produced.

\subsection{Error control}

Because of the low quality and/or quantity of DNA derived from noninvasive genetic samples, genotyping errors are difficult to eliminate and may lead to incorrect genotypes and consequently to overestimated population size [24]. To avoid erroneous results arising out of genotyping error caused by nonreplicable results or the nonamplification of an allele in heterozygotes ('allelic dropout'), all results were subject to the appropriate amount of replication given the quantity of DNA present as detailed by Arandjelovic et al. [22]. However, such a 'multitube' approach cannot detect errors that may be created when scoring or transcribing data into a database [25]. We therefore also used two screening methods, the examining bimodality and difference in capture history tests, implemented in the DROPOUT package to detect genotyping errors [26]. We also used MICROCHECKER to look for genotyping errors owing to null al- leles, short allele dominance (large allele dropout) and scoring errors because of stuttering [27].

\subsection{Genotype data analysis}

We used CERVUS 3.0 software [28] to find matching genotypes and to assess the probability of full siblings or unrelated individuals having an identical multilocus genotype $\left(\mathrm{PID}_{\text {sib }}\right.$ and PID). To determine with $99.9 \%$ confidence that two matching samples originated from the same individual, we determined the minimum number of loci necessary to obtain a $\mathrm{PID}_{\text {sib }}$ value of $\leqslant 0.001$ [29]. Matching samples were given a consensus ID and genotype for use in subsequent analysis. Genotypes from different samples mismatching at three or fewer loci were re-examined for possible scoring or transcribing data errors. CERVUS 3.0 was also used to calculate the number of alleles per locus $(A)$, observed $\left(H_{\mathrm{O}}\right)$ and expected $\left(H_{\mathrm{E}}\right)$ heterozygosities for each locus, and to test for deviations of used loci from HardyWeinberg equilibrium. Genotypic disequilibrium between loci within the population was tested using FSTAT version 2.9.3.2 [30]. Significance values were adjusted by Bonferroni correction for multiple testing, as implemented in the software.

\subsection{Population size estimation}

Grouping all samples into a single-sampling session scheme, we then calculated genetic capture-recapture estimates in 3 models. Miller et al. [31] developed a maximum likelihood estimator implemented in the CAPWIRE software. Unlike the traditional mark-recapture method, this method deals efficiently with data inferred from multi-observations of individuals within a sampling session. The even capture model (ECM) assumes there is no capture heterogeneity in the data set, whereas the two innate rates model (TIRM) assigns individuals as having unequal capture probability. We used both ECM and TIRM models to estimate population size, as we could not be certain whether capture heterogeneity existed in our data. Both models assume a closed population (no births, deaths, or migration) and a recapture probability equaling the capture probability. Miller et al. [31] found one heterogeneity estimator in the program CAPTURE $\left(\mathrm{M}_{\mathrm{h}}\right.$-Chao) [32] performed much better when dealing with populations larger than 100 individuals. Given our expected large census population size, we also used $\mathrm{M}_{\mathrm{h}}$-Chao to estimate population size. The $\mathrm{M}_{\mathrm{h}}$-Chao Model is implemented in the program CAPTURE [33]. Algorithms in CAPTURE also assume the population is closed to births, deaths, emigration and immigration. All of the three models yield population size estimates and a $95 \% \mathrm{CI}$ of this estimate.

\subsection{Birth data collection}

Birth data were collected from a provisioned free-ranging 
group at the DLT locality between 2006 and 2010 . The individual monkeys in the DLT group were provided with food from 2005 so that we could achieve better observation. Apples, chopped radishes, lichen and peanuts were scattered on the ground randomly three times per day $(9: 00,12: 00$, and 16:30). To minimize the effect of artificial feeding on their natural diet, we controlled the quantity of provisioning for each monkey. Individuals in this group were accustomed to the approach of researchers. Our observations were performed at distances between 0.5 and $50 \mathrm{~m}$. Observations from such close distance enabled us to identify each individual and to distinguish the sex of the new-born infants.

\section{Results}

We attempted to genotype 337 DNA extracts at 16 loci. Nine extracts did not yield any genotypes, 15 extracts yielded genotypes at fewer than 5 loci, and 26 extracts yielded genotypes at 5 to 10 loci. Among the remaining 287 extracts, 196 (68.3\%) extracts provided a complete genotype at the 16 loci. The DNA concentration of most unsuccessful extracts was low $(<35 \mathrm{pg} / \mathrm{uL})$. The locus D13S321 deviated significantly from the Hardy-Weinberg equilibrium. After applying Bonferroni correction, none of the paired loci significantly deviated from genotypic disequilibrium. Overall, the microsatellite loci used were polymorphic with an average of 4.5 alleles. The average observed and expected heterozygosity of 16 loci was 0.591 and 0.589 , respectively (Table 1 ). The probability of two full siblings sharing the same multilocus genotype at the 16 loci was $2.11 \times 10^{-5}$ (PIDsib) and the corresponding probability for two unrelated individuals was $2.71 \times 10^{-11}$.

Table 1 Characteristics of microsatellite loci used in this study ${ }^{\text {a) }}$

\begin{tabular}{llccc}
\hline \multicolumn{1}{c}{ Locus } & $A$ & $N$ & $H_{\mathrm{O}}$ & $H_{\mathrm{E}}$ \\
\hline D14S306 & 4 & 153 & 0.575 & 0.590 \\
D7S2204 & 6 & 151 & 0.728 & 0.699 \\
D5S1457 & 6 & 148 & 0.601 & 0.638 \\
D6S1056 & 5 & 149 & 0.497 & 0.507 \\
D1S1656 & 5 & 150 & 0.660 & 0.629 \\
D6S493 & 4 & 151 & 0.702 & 0.695 \\
D10S611 & 5 & 148 & 0.669 & 0.718 \\
D6S474 & 3 & 150 & 0.633 & 0.578 \\
D1S1665 & 4 & 146 & 0.610 & 0.653 \\
D2S442 & 7 & 140 & 0.750 & 0.742 \\
D10S1432 & 7 & 150 & 0.673 & 0.661 \\
D1S533 & 3 & 152 & 0.592 & 0.569 \\
D7S817 & 3 & 152 & 0.507 & 0.441 \\
D7S1826 & 3 & 151 & 0.377 & 0.333 \\
D10S676 & 3 & 152 & 0.572 & 0.549 \\
D13S321 & 4 & 143 & 0.315 & 0.421 \\
Mean & 4.5 & 149 & 0.591 & 0.589 \\
\hline
\end{tabular}

a) $A$, Number of alleles per locus; $N$, number of samples; $H_{\mathrm{O}}$, observed heterozygosity; $H_{\mathrm{E}}$, expected heterozygosity.
MICRO-CHECKER analysis provided no evidence of null alleles, large allele dropout or scoring error because of stuttering in our final data set, with the exception of D13S321, which showed evidence of null alleles and possible scoring error causd by stuttering. DROPOUT analysis indicated genotyping errors were reduced to a nonsignificant level.

Among the 287 extracts that yielded genotypes, 153 unique individuals were identified, which represented the minimum number of individuals using the area. The number of captures/recaptures per individual ranged from 1 to 3 . The ECM produced the lowest point estimate of 934 (95\% $\mathrm{CI}_{\mathrm{ECM}}$ : 603-1484) monkeys, whereas the TIRM gave the highest point estimate of 1044 monkeys and the smallest confidence interval (95\% CI TIRM: 613-1409). The $\mathrm{M}_{\mathrm{h}}$-Chao model gave an intermediate point estimate of 970 monkeys and the largest confidence interval (95\% CI M $\mathrm{M}_{\mathrm{h}}$-Chao: 5831704). Considering the entire $705 \mathrm{~km}^{2}$ study area, the density of $R$. roxellana in the SNR was estimated to be 1.32 monkeys $/ \mathrm{km}^{2}$ (95\% CI: $0.86-2.1$ ) with the ECM, 1.48 monkeys $/ \mathrm{km}^{2}$ (95\% CI: 0.87-2) with the TIRM, and 1.38 monkeys $/ \mathrm{km}^{2}$ (95\% CI: 0.83-2.42) with the $\mathrm{M}_{\mathrm{h}}$-Chao model.

Most monkeys were genetically sampled ('captured') only once, but 11 monkeys were sampled twice and one monkey was sampled 3 times. One individual was sampled at 3 different localities (XLT, JHL, and SBJ) and an additional 6 monkeys were samples at both XLT and JHL. Three individuals were sampled at both XLT and SBJ, and 2 at both SBJ and YJW. Given that groups of $R$. roxellana were relatively stable and not characterized by periods of fission and fusion [34], our results suggested that the individuals sampled at those 4 sites (JHL, YJW, XLT, and SBJ) belong to the same group. These collection localities span the road in the reserve, and so further indicate that $R$. roxellana were able to cross the road. One additional individual was identified at both the DR and DW sampling localities, but additional sampling would be necessary to estimate the composition of additional groups.

Five extracts failed to amplify using the sexing assay. Among the remaining 148 samples we identified 91 females and 57 males. The sex ratio $(\mathrm{M} / \mathrm{F})$ for all individuals was about 1:1.6. For the DLT group, 36 infants (17 males and 19 females) were born from 2006 to 2010 . The sex ratio (M/F) in this group was approximately $1: 1.12$ (Table 2).

Table 2 Sex of new-born infants and sex ratio in the DLT group from 2006 to 2010

\begin{tabular}{cccc}
\hline \multirow{2}{*}{ Year } & \multicolumn{2}{c}{ Sex of new-born infants } & \multirow{2}{*}{ Sex ratio (M:F) } \\
\cline { 2 - 3 } & Male & Female & $1: 2$ \\
\hline 2006 & 2 & 4 & $1: 0.67$ \\
2007 & 3 & 2 & $1: 1$ \\
2008 & 6 & 6 & $1: 0.67$ \\
2009 & 3 & 2 & $1: 1.67$ \\
2010 & 3 & 5 & $1: 1.12$ \\
Total & 17 & 19 & \\
\hline
\end{tabular}




\section{Discussion and conclusions}

Small and island populations have a higher extinction risk than mainland populations [35]. However, direct estimates of extinction risk are frequently unavailable. O'Grady et al. [36] proposed that population size was the best predictor of extinction risk and was therefore the most cost-effective data to collect on threatened species. Population monitoring is essential to evaluate the success of conservation efforts, yet standard survey methods may be ineffective because animals flee silently before they are seen [15]. Population estimation using a noninvasive genetic census technique does not require visual or physical contact with the individuals, and allow for larger sample sizes to be collected than would be possible with traditional trapping methods [24]. However, several sources of potential error are associated with this method, including failure to identify individuals and incorrect assignment of individual genotypes [24]. Population estimates have tended to be biased downward because of the 'shadow effect' [37], a phenomenon of multiple individuals showing identical tags caused by using too few loci or loci with low heterozygosity. In this study, the set of 16 microsatellite loci was enough to differentiate between the individuals in the area. Therefore, the shadow effect was not a serious problem in our study. Overestimation because of genotyping errors is much more difficult to control. In this study, we used a two-step multiplex PCR method, which can significantly increase the success rate and efficiency of genotyping and substantially reduce the average allelic dropout rates [22]. Analysis with DROPOUT also indicated genotyping errors in our study decreased to a negligible level and did not affect the estimate of population size.

This study demonstrated the utility of genetic capturerecapture to estimate the size of $R$. roxellana population. All of the 3 models gave consistent point estimates. However, the ECM model is known to be biased downwards when capture heterogeneity exists [6]. Therefore the $\mathrm{M}_{\mathrm{h}}$-Chao and TIRM models might provide the most robust estimates. Our results indicated the population size of $R$. roxellana in the SNR substantially increased from 500 to 1044 individuals during the past two decades. Based on a direct count, Yang et al. [38] estimated the SNR population size to be 1282 individuals, a value larger than our estimates but still within our $95 \%$ CI. Because of the preliminary nature and limited sampling effort in the present study, we could only obtain a rough estimate of population size and a wide confidence interval. Previous studies have shown that the sampling intensity influences the confidence of the estimate [31]. The difficulty of finding $R$. roxellana in the field is the major reason we were unable to collect additional fecal samples in this study. $R$. roxellana usually travel to remote and inaccessible areas rendering it difficult for researchers to locate them. For instance, the QJP group was untraced for more than 6 months in 2008. In addition, because of the small population size, it took considerable searching time to lo- cate monkeys in such a large area. However, the focus in this study was not to obtain a precise estimate of population size but to elucidate the population trend. Thus, sampling effort can be reduced to the point that will provide enough statistical power to detect the trend [6]. To achieve an accurate estimate of population size in future, the number of samples collected should be approximately three times the 'assumed' number of individuals [6].

Sex ratio is also a key parameter to monitor population health, because it can dictate mate competition/choice and affect population growth rates [39]. In the field, it is very difficult to distinguish the sex of infant and subadult $R$. roxellana (subadult males: 3-5 years old, subadult females: 3-4 years old, [34]). Therefore, previous field studies only described the sex ratio of adults, while in the present study we do not know the ages of the monkeys and so report the sex ratio for all sampled individuals. For the SNR population, the adult sex ratio $(\mathrm{M} / \mathrm{F})$ differed in previous studies from $1: 1.42$ in ref. [4] and $1: 2.8$ in ref. [34]. The sex ratio $(\mathrm{M} / \mathrm{F})$ of all individuals of $1: 1.6$ in the present study was similar to that previously reported for adults, which showed a bias toward females. Qi et al. [40] found slightly more male infants than female were born based on four years of investigation in the Qinling mountains. However, observation of the DLT group for five years suggests that in SNR the sex ratio of new-born infants does not significantly differ from $1: 1(\mathrm{M} / \mathrm{F}=1: 1.12)$ (Table 2). There are at least two possible explanations for our observed female-biased sex ratio in the SNR. One possibility is that the social structure of $R$. roxellana resulted in the collection of a biased set of samples. $R$. roxellana groups are composed of two basic units: one male units (OMUs) and all male units (AMUs). An OMU is composed of one adult male, several adult females, several subadults and infants. An AMU contains several adult and subadult males. The sex ratio (M/F) in OMUs was estimated previously as 1:7 and so is highly biased toward females [34]. Field investigations showed the individuals in AMUs are consistently located at the periphery of the group whenever resting or moving [34]. This spatial distribution of individuals can result in unequal capture possibilities for the two sexes. The female-biased sex ratio suggests we collected more samples from OMUs than from AMUs. An alternative, nonmutually exclusive explanation for our results is that the population genuinely exhibits a female-biased sex ratio. Deviations from an equal sex ratio are common in mammalian populations and, according to a complex framework of models and hypotheses, either adaptive mechanisms (social or parental control of sex ratio from conception onward $[41,42]$ ) or nonadaptive ones, e.g., unpredictable environmental events leading to different mortality rates in the sexes $[43,44]$, can shift primate sex ratios from parity [45]. Future work employing a more extensive genetic census of the population will serve to refine the estimate of the population sex ratio.

Natural and anthropogenic landscape features, such as 
rivers, mountain ranges and roads, can provide complete or semipermeable barriers that alter animal dispersal paths and movement patterns [46]. Roads have been shown previously to affect animal movement patterns and typically impede dispersal and reduce gene flow [47]. In the present study, we showed that the heavy traffic road did not present a barrier to movement of $R$. roxellana groups, which is probably because of the relatively high mobility of $R$. roxellana.

The Sichuan snub-nosed monkey is a flagship species for conservation in China, and is an object of public interest and national pride. Protection of Sichuan snub-nosed monkeys as 'umbrella' populations is both a biologically and culturally effective way to preserve whole ecosystems [48]. Although the population experienced a slight increase during the past two decades, the outlook for the species is still worrisome. In the winter of 2008 , the worst snowfall in the last 5 decades hit large portions of southern and central China [49]. The huge storm and extremely low temperature killed 73 individuals in one group, a reduction of $27.2 \%$ [50], an impact consistent with the high vulnerability of island populations to extreme environmental changes. Reed et al. [51] defined minimum viable population sizes (MVPs) that could ensure a $99 \%$ probability of population persistence for 40 generations. These authors estimated the mean MVPs to generally be 7316 adults, an estimate that was far higher than the current size of the SNR $R$. roxellana population. Given the small population size and vulnerability to environmental changes, we suggest that this population merits special concern. To ensure long-term population survival, it is imperative to facilitate gene flow among groups and stop deforestation and fragmentation of habitats.

We thank Dr. Katerina Guschanski for experimental guidance. We also thank Grit Schubert, Mimi Arandjelovic, Kevin Langergraber, and Jyotsna Bhagavatula for helpful discussions. This work was supported by the Key Project of National Natural Science Foundation of China (31130061), the Project of Public Benefit (201104073), and the Max Planck Society.

1 Jablonski N G. The response of Catarrhine primates to Pleistocene environmental fluctuations in east Asia. Primates, 1998, 39: 29-37

2 Ren R M, Su Y J, Yan K H, et al. Preliminary survey of the social organization of Rhinopithecus roxellana in Shennongjia National Nature Reserve, Hubei, China. In: Jablonski N G, ed. The Natural History of the Doucs and Snub-nosed Monkeys. Singapore: World Scientific, 1998. 269-277

3 Li B G, Pan R L, Oxnard C E. Extinction of snub-nosed monkeys in China during the past 400 years. Int J Primatol, 2002, 23: 1227-1244

4 Zhu Z Q. The ecological study of sichuan snub-nosed monkeys (Rhinopithecus roxellana) in Shennongjia Nature Reserve (in Chinese). Hubei Forestry Sci Technol, 2003, Z1: 46-52

5 Quan G Q, Xie J Y. Research on the Golden Monkey (in Chinese). Beijing: Science and Education Publishing House, 2002

6 Puechmaille S J, Petit E J. Empirical evalution of non-invasive capture-mark-recapture estimation of population size based on a single sampling session. J Appl Ecol, 2007, 44: 843-852

7 Kohn M H, York E C, Kamradt D A, et al. Estimating population size by genotyping faeces. Proc R Soc B, 1999, 266: 657-663

8 Eggert L S, Eggert J A, Woodruff D S. Estimating population sizes for elusive animals: The forest elephants of Kakum National Park, Ghana. Mol Ecol, 2003, 12: 1389-1402
9 Bellemain E V A, Swenson J E, Tallmon D, et al. Estimating population size of elusive animals with DNA from hunter-collected feces: Four methods for brown bears. Conserv Biol, 2005, 19: 150-161

10 Zhan X J, Li M, Zhang Z, et al. Molecular censusing doubles giant panda population estimate in a key nature reserve. Curr Biol, 2006, 16: $451-452$

11 Arrendal J, Vilà C, Björklund M. Reliability of noninvasive genetic census of otters compared to field censuses. Conserv Genet, 2007, 8: 1097-1107

12 Guschanski K, Vigilant L, McNeilage A, et al. Counting elusive animals: Comparing field and genetic census of the entire mountain gorilla population of Bwindi Impenetrable National Park, Uganda. Biol Conserv, 2009, 142: 290-300

13 Arandjelovic M, Head J, Kühl H, et al. Effective non-invasive genetic monitoring of multiple wild western gorilla groups. Biol Conserv, 2010, 143: 1780-1791

14 Vigilant L, Guschanski K. Using genetics to understand the dynamics of wild primate populations. Primates, 2009, 50: 105-120

15 Savage A, Thomas L, Leighty K A, et al. Novel survey method finds dramatic decline of wild cotton-top tamarin population. Nat Commun, 2010, 1: 30-36

16 Cui L W, Zhong T, Xiao L, et al. Group size and composition of black-and white snub-nosed monkey (Rhinopithecus bieti) estimated by faeces of sleeping sites at Baima Snow Mountain. Zool Res, 2006, 27: 337-343

17 Zhu Z Q. Status and conservation of snub-nosed monkey at Shennongjia Nature Reserve (in Chinese). Chin Wildl, 1992, 67: 6-17

18 Li Y M. The effect of forest clear-cutting on habitat use in Sichuan snub-nosed monkey (Rhinopithecus roxellana) in Shennongjia Nature Reserve, China. Primates, 2004, 45: 69-72

19 Li Y M. The seasonal daily travel in a group of Sichuan snub-nosed monkey (Pygathrix roxellana) in Shennongjia Nature Reserve, China. Primates, 2002, 43: 271-276

20 Nsubuga A M, Robbins M M, Roeder A D, et al. Factors affecting the amount of genomic DNA extracted from ape feces and the identification of an improved sample storage method. Mol Ecol, 2004, 13: 2089-2094

21 Morin P A, Chambers K E, Boesch C, et al. Quantitative PCR analysis of DNA from noninvasive samples for accurate microsatellite genotyping of wild chimpanzees (Pan troglodytes verus). Mol Ecol, 2001, 13: 2089-2094

22 Arandjelovic M, Guschanski K, Schubert G, et al. Two-step multiplex polymerase chain reaction improves the speed and accuracy of genotyping using DNA from noninvasive and museum samples. Mol Ecol Resour, 2009, 9: 28-36

23 Villesen P, Fredsted T. A new sex identification tool: One primer pair can reliably sex ape and monkey DNA samples. Conserv Genet, 2006, 7: 455-459

24 Waits J L, Leberg P L. Biases associated with population estimation using molecular tagging. Anim Conserv, 2000, 3: 191-199

25 Schwartz M K, Cushman S A, McKelvey K S, et al. Detecting genotyping errors and describing American black bear movement in northern Idaho. Ursus, 2006, 17: 138-148

26 McKelvey K, Schwartz M K. DROPOUT: A program to identify problem loci and samples for noninvasive genetic samples in a capture-mark-recapture framework. Mol Ecol Notes, 2005, 5: 716-718

27 Van Oosterhout C V, Hutchinson W F, Wills D P M, et al. MICROCHECKER: Software for identifying and correcting genotyping errors in microsatellite data. Mol Ecol Notes, 2004, 4: 535-538

28 Kalinowski S T, Taper M L, Marshall T C. Revising how the computer program CERVUS accommodates genotyping error increases success in paternity assignment. Mol Ecol, 2007, 16: 1099-1106

29 Waits P L, Luikart G, Taberlet P. Estimating the probability of identity among genotypes in natural populations: Cautions and guidelines. Mol Ecol, 2001, 10: 249-256

30 Goudet J. FSTAT, a program to estimate and test gene diversities and fixation indices (version 2.9.3.2). 2001. Available from http: //www. unil.ch/izea/softwares/fstat.html.

31 Miller C R, Joyce P, Waits L P. A new method for estimating the size 
of small populations from genetic mark-recapture data. Mol Ecol, 2005, 14: 1991-2005

32 Chao A. Estimating animal abundance with capture frequency data. J Wildl Manage, 1988, 52: 295-300

33 Otis D L, Burnham K P, White G C, et al. Statistical inference from capture data on closed animal populatons. Wildl Monogr,1978, 62

34 Ren R M, Yan K H, Su Y J, et al. The Society of Golden Monkeys (Rhinopithecus roxellana) (in Chinese). Beijing: Beijing University Press, 2000

35 Frankham R. Inbreeding and extinction: island populations. Conserv Biol, 1998, 12: 665-675

36 O'Grady J J, Reed D H, Brook B W, et al. What are the best correlates of predicted extinction risk? Biol Conserv, 2004, 118: 513-520

37 Mills L S, Citta J J, Lair K P, et al. Estimating animal abundance using noninvasive DNA sampling: Promise and pitfalls. Ecol Appl, 2000, 10: 283-294

38 Yang J Y, Liao M Y, Yu H L, et al. Protection and research status of golden monkeys in Shennongjia (in Chinese). World Sci-tech R\&D, 2008, 30: 418-421

39 Emlen S T, Oring L W. Ecology, sexual selection and the evolution of mating systems. Science, 1977, 197: 215-223

40 Qi X G, Li B G, Ji W H. Reproductive parameters of wild female Rhinopithecus roxellana. Am J Primatol, 2008, 70: 311-319

41 Trivers R L, Willard D E. Natural selection of parental ability to vary sex ratio of offspring. Science, 1973, 179: 90-92

42 van Schaik C P, van Noordwijk M A. Social stress and the sex ratio of neonates and infants among non-human primates. Neth J Zool, 1983, 33: 249-265

43 Bonefant C, Gaillard J M, Loison A, et al. Sex-ratio variation and reproductive costs in relation to density in a forest-dwelling population of red deer (Cervus elaphus). Behav Ecol, 2003, 14: 862-869

44 Kruuk L E B, Clutton-Brock T H, Albon S D, et al. Population density affects sex variation in red deer. Nature, 1999, 399: 459-461

45 Norscia I, Palagi E. Berenty 2006: Census of Propithecus verreauxi and possible evidence of population stress. Int J Primatol, 2008, 29: 1099-1115

46 Long E S, Diefenbach D R, Wallingford B D, et al. Influence of roads, rivers, and mountains on natal dispersal of White-tailed deer. $\mathrm{J}$ Wildl Manage, 2010, 74: 1242-1249

47 Trombulak S C, Frissell C A. Review of ecological effects of roads on terrestrial and aquatic communities. Conserv Biol, 2000, 14: 18-30

48 Kirkpatrick R C. The natural history and conservation of the snub-nosed monkeys (genus Rhinopithecus). Biol Conserv, 1995, 72: 363-369

49 Stone R. Ecologists report huge storm losses in China's forests. Science, 2008, 319: 1318-1319

50 Li Y M, Liu X C, Liao M Y, et al. Characteristics of a group of Hubei golden snub-nosed monkeys (Rhinopithecus roxellana hubeiensis) before and after major snow storms. Am J Primatol, 2009, 71: 1-4

51 Reed D H, O'Grady J J, Brook B W, et al. Estimates of minimum viable population sizes for vertebrates and factors influencing those estimates. Biol Conserv, 2003, 113: 23-34

Open Access This article is distributed under the terms of the Creative Commons Attribution License which permits any use, distribution, and reproduction in any medium, provided the original author(s) and source are credited. 